\title{
National Pharmacy Pain Management Program at Ministry of Health in Saudi Arabia
}

\author{
Yousef Ahmed Alomi* \\ National Clinical Pharmacy, and Pharmacy Practice Programs, Head, Pharmacy R \& D Administration Ministry of Health, Saudi Arabia
}

Submission: June 14, 2016; Published: June 29, 2017

*Corresponding author: Yousef Ahmed Alomi,The Past General Manager of General Administration of Pharmaceutical Care, Head, National Clinical Pharmacy, and Pharmacy Practice Programs, Head, Pharmacy R \& D Administration, Ministry of Health, P.0.BOX 100, Riyadh 11392, Riyadh, Saudi Arabia, Tel: +966504417712; Email: yalomi@gmail.com

\begin{abstract}
Over short of time, the General Administration of Pharmaceutical Care established several pharmacy practice programs based on patient demand, preventing drug misadventures, and poor health care provider's knowledge and perception and national and international hospital pharmacy accreditation's requirements. National pain management program was among them. The system initially started in the 2000 s in Saudi Arabia, and the first program at Ministry of Health hospital stated in 2008, and then become national program in 2013. The program had astrategic plan, and policy and procedures, with Key performance indication to measure the implementation of the program. The program prevents drug misuse and avoid additional economic burden on health care system in Saudi Arabia
\end{abstract}

Keyword: Pain Management; Pharmaceutical care; Pharmacy; Ministry of Health; Saudi Arabia

\section{Introduction}

The history of starting pain management services in Saudi Arabia is not clear. However, may be the first hospital started the program at Armed forced hospital in 1994. The clinical audit study from 1994-2003 and more 10,000 patients described the acute pain services at the hospital [1]. The pain management clinic established at Jeddah teaching hospital in January 2000 and provided the services through the year of 2004 [2]. In 2008, the hospital director of the biggest hospital at Ministry of Health (1400 beds) assigned the author as a head of regional drug information center. The author established the infrastructure of all clinical services including but not limited to the following poising control services, anticoagulation program, pharmacokinetics services, oncology pharmacy services, Surgical and Orthopedic pharmacy services, pharmacoeconomic program, and pain management program [3]. The pain management program activated at the same hospital in 2010. The program consisted of goal and objectives, the job description of a pain management clinical pharmacist, policy and procedures of pain management services, pain management guidelines. The local hospital committee founded and headed by the director of aesthetics department, coordinated by trained pain management clinical pharmacist, and membership representative of the surgical, medical department, pediatrics development, nursing department, and quality management departments. The pain management guidelines designed as physician order form; both adult acute, and chronicpain management. Each type of pain treatment list medication based on $\mathrm{MOH}$ formulary. The options list made as first-line therapy, second choice, and last line therapy.

The medications chosenbased on update guidelines of adult's pain management, updated literature and pharmacoeconomic based. If the treating doctors wish to choose another option, he has to write the justification on that. All physician formats approved by a local hospital of pain management. Also, the committee formulated the pain management team contained anesthesiologist, nurse, and pain management clinical pharmacist. They do daily around five days per a week for postoperative patients, and consultation received from different departments at the hospital. The pharmacist should follow the adherence of the program, recommended the appropriate drug therapy based individual patient, and follow up and document any drug-related problems, thus correct them accordingly. Measurement patient quality of life and a cost impact required from clinical pharmacist. The program continued until 2012. 


\section{Pharmacy Pain Management Program}

The demand for pain management program is very high in Kingdom of Saudi Arabia. The program required for hospital accreditation process at national and international accreditation institutions [4-5]. The pain management medication is underutilization in Saudi Arabia. In a study at teaching hospital in Riyadh city, the authors found with 160 oncologic patients $40 \%$ of whose suffered from pain not given any medication for pain while more $50 \%$ of then received inappropriate painkiller [6]. Also, the background of medical doctor or pharmacists or nurses showed poor knowledge and negative perception toward pain management medication during studentship [7-9]. The author not familiar with any published literature at in Saudi Arabia, and Gulf countries or the Middle East described national pharmacy pain management program

\section{National Pharmacy Pain Management Program in Kingdom Of Saudi Arabia}

In late 2012, the author promoted to General Manager of Pharmaceutical care. The author and his administration revised the general plan of MOH 2010-2020, updated the general plan of pharmaceutical care, and founded more thirty pharmacy practices and clinicalpharmacy program. Pain management services were one of them [10-13]. The author established a new central committee of pain management consisted of representing twenty regions over all the kingdom of Saudi Arabia. The committee updates all policy and procedures of previous hospital pain management, update the physician orders format, all related thing of the program. The member shared in designing Saudi clinical guidelines treatment of skill cell disease. The central committee established a plan for next five years as explored in (Table 1). The central committee started strategic plan over a five-year period, and the program beganwith nine hospitals then expanded to different regions as explored in (Table 2). The central committee founded key performance indicators to follow up the implementation of the program as shown in (Table 3), and the effective day of applying pain management at all MOH health care institution was on 4 September 2012 by a memorandum sent by General Manager of Pharmaceutical care.

Table 1: Type of Pharmacy pain management committees.

\begin{tabular}{|c|c|c|c|c|}
\hline & Membership & Functions & $\begin{array}{c}\text { Frequently } \\
\text { meeting }\end{array}$ & Reporting \\
\hline \multicolumn{5}{|c|}{ Central Pharmacy Pain Management Committee } \\
\hline 1 & $\begin{array}{l}\text { 1. National Clinical Pharmacy as head } \mathrm{f} \text { the } \\
\text { committee } \\
\text { 2. Pain management clinical pharmacist } \\
\text { coordinator } \\
\text { 3. Pain management clinical pharmacist from } \\
\text { each twenty regions }\end{array}$ & $\begin{array}{l}\text { 1. Establish of national pharmacy pain management program at } \\
\text { MOH hospitals } \\
\text { 2. Setup strategic planning of national pharmacy pain } \\
\text { management } \\
\text { stewardship program at MOH institutions } \\
\text { 3. Follow up and update of national pharmacy pain management } \\
\text { stewardship programat MOH hospitals } \\
\text { 4. Implement and Follow-upnational pharmacy pain } \\
\text { management stewardship committee at MOH hospitals } \\
\text { 5. Setup up policy and procedures of national pharmacy pain } \\
\text { management } \\
\text { stewardship program at MOH institutions } \\
\text { 6. Setup and implement national pharmacy pain management } \\
\text { stewardship program key performance indicators at MOH } \\
\text { institutions } \\
\text { 7. Review all reports of national pharmacy pain management } \\
\text { stewardship program from all MOH institutions }\end{array}$ & Monthly & $\begin{array}{l}\text { Every } \\
\text { three } \\
\text { months }\end{array}$ \\
\hline \multicolumn{5}{|c|}{ Hospital Pain Management Committee } \\
\hline 2 & $\begin{array}{l}\text { 1. Consultant Anesthesiology or Intensivistor } \\
\text { chief of pharmacy as head of the committee } \\
\text { 2. Internal Medicine Physician } \\
\text { 3. Surgeon } \\
\text { 4. Emergency physician } \\
\text { 5. Pediatrician } \\
\text { 6. MD of obstetrics and gynecology } \\
\text { 7. Nurse } \\
\text { 8. Chief of Clinical Pharmacy Department } \\
\text { 9. Drug Information Center pharmacist } \\
\text { 10. Psychologist } \\
\text { 11. Physiotherapist } \\
\text { 12. Social Service }\end{array}$ & $\begin{array}{l}\text { 1. Supervise the implementation of pain management program } \\
\text { at the hospital } \\
\text { 2. Supervision of program activation, program follow-up, } \\
\text { program } \\
\text { Evaluation, and program development at the hospital. } \\
\text { 3. Approval of the pain management team members } \\
\text { 4. Review and update pain management protocol at least } \\
\text { annually } \\
\text { 5. Review the job description, policy, and procedures of pain } \\
\text { management team } \\
\text { 6. Supervising of pain management clinic } \\
\text { 7. Examine the pain program needs of equipment and medical } \\
\text { supplies,...etc } \\
\text { 8. Submit reports on the progress of the Commission's work and } \\
\text { the team and its effectiveness for patients and health team every } \\
\text { three months central pain management committee }\end{array}$ & Monthly & $\begin{array}{l}\text { Every } \\
\text { three } \\
\text { months }\end{array}$ \\
\hline
\end{tabular}


Pain Management team

The team consists of

- From an Anesthesiology or intensive care or pain management clinical pharmacist.

- Pain management clinical pharmacist pain

- Pain Management Nurse pain.

3

The pain management program begins with postoperative patients. All other types of pain at the request of the consulting pain management team. The services expand coverage to all patients in the institutions, and maybe need more than one team to cover patients as needed.
The daily round all patients suffering from pain. Operate hospital pain clinics.

Provide counseling for pain management.

Apply the pain management protocol at hospital

Follow up the pain management patient and resolve thedrug related problem

Measure patient outcomes, quality of life, and cost avoidance

Receive any pain management consultation from any treating teams at the hospitals

Daily

Monthly

7. Receive any pain man

Table 2: Strategic plan of pharmacy pain management program.

\begin{tabular}{|c|c|c|}
\hline & Elements of the plan & \\
\hline \multicolumn{3}{|l|}{2014} \\
\hline Stage 1 & $\begin{array}{l}\text { Establish the central national pharmacy pain management Committee } \\
\text { Central at Ministry of Health } \\
\text { Set the national pharmacy pain management Committee at twenty Region } \\
\text { Set the national pharmacy pain management Committee at Peripheral } \\
\text { ninety Hospital } \\
\text { Implement of national pharmacy pain management program at ninety } \\
\text { Hospital } \\
\text { Establish the adults pain management physician order sheet booklet and } \\
\text { distribute it to all hospitals }\end{array}$ & $\begin{array}{l}\text { Establish the adults Pain Management physician order } \\
\text { as computerized physician order entry (CPOE)with } \\
\text { implementation at fifty hospitals } \\
\text { Deliver the pharmacy pain management training Courses } \\
\text { at MOH } \\
\text { Deliverthe pharmacy pain management courses for } \\
\text { twenty regions }\end{array}$ \\
\hline \multicolumn{3}{|l|}{2015} \\
\hline Stage 2 & $\begin{array}{l}\text { Follow-upthe hospital pain management committee at ninety Hospital } \\
\text { Follow up the implementation of pain management program with previous } \\
\text { ninety Hospital } \\
\text { Establish of pain management committee at new ninety Hospital } \\
\text { Design pediatrics pain management physician order sheet booklet and } \\
\text { distribute it to all hospitals } \\
\text { Design the Pediatricspain management physician order as CPOE and apply } \\
\text { it at fifty hospitals }\end{array}$ & $\begin{array}{l}\text { Revise and update adults pain management physician } \\
\text { order sheet booklet and distributed to all hospitals } \\
\text { Revise and update adults pain management physician } \\
\text { order as CPOE and apply it at fifty hospitals } \\
\text { Review of adults pain management guidelines utilization } \\
\text { Review adults pain management medications } \\
\text { consumption } \\
\text { Deliver the adults pain management training Courses at } \\
\text { MOH } \\
\text { Deliver the adults pain management training courses for } \\
\text { twenty regions }\end{array}$ \\
\hline \multicolumn{3}{|l|}{2016} \\
\hline Stage 3 & $\begin{array}{l}\text { Follow-up of Pain Management Committee at Peripheral previous } 180 \\
\text { Hospital } \\
\text { Follow up the Implementation of Pain Management program at previous } \\
180 \text { Hospitals } \\
\text { Establish of pain management committee at new ninety hospitals } \\
\text { Revise and update adults and pediatrics pain management physician order } \\
\text { sheet booklet and distribute it to all hospitals }\end{array}$ & $\begin{array}{l}\text { Revise and update adults and pediatrics pain } \\
\text { management physician order as CPOE and apply it at fifty } \\
\text { hospitals } \\
\text { Review of Adults and Pediatrics pain management } \\
\text { guidelines utilization } \\
\text { Review Adults and Pediatrics pain management } \\
\text { medications consumption } \\
\text { Delivery of Adults and Pediatrics pain management } \\
\text { training Courses at MOH } \\
\text { Delivery of Adults and Pediatrics pain management } \\
\text { training courses for twenty regions }\end{array}$ \\
\hline \multicolumn{3}{|l|}{2017} \\
\hline Stage 4 & $\begin{array}{l}\text { Follow-up of pain management committee at previous } 270 \text { Hospital } \\
\text { Follow up the Implementation of pain management program at previous } \\
270 \text { Hospital } \\
\text { Establish of Adults and Pediatricspain management committee and } \\
\text { program at new peripheral ninety private Hospitals } \\
\text { Revise and update adults and pediatrics pain management physician order } \\
\text { sheet booklet and distribute it to all hospitals } \\
\text { Revise and update adults and pediatrics pain management physician order } \\
\text { as CPOE and apply it at fifty hospitals }\end{array}$ & $\begin{array}{l}\text { Review of Adults and Pediatrics pain management } \\
\text { guidelines utilization } \\
\text { Review Adults and Pediatrics pain management } \\
\text { medications consumption } \\
\text { Deliverthe Adults and Pediatrics pain management } \\
\text { training Courses at MOH and private sector } \\
\text { Deliverthe Adults and Pediatrics pain management } \\
\text { training courses for twenty regions and private sector }\end{array}$ \\
\hline
\end{tabular}




\begin{tabular}{|c|c|c|}
\hline 2018 & & \\
\hline Stage 5 & $\begin{array}{l}\text { Follow-up of pain management committee at peripheral previous } 270 \\
\text { MOH hospital and } 90 \text { private hospitals } \\
\text { Follow up the implementation of pain management program at previous } \\
270 \text { hospital and } 90 \text { private hospitals } \\
\text { Establish of Adults and Pediatricspain management committee and } \\
\text { program at new peripheral ninety private Hospitals } \\
\text { Revise and update adults and pediatrics Pain Management physician order } \\
\text { sheet booklet and distribute it to all MOH hospitals and private sector }\end{array}$ & $\begin{array}{l}\text { Revise and update adults and pediatrics pain } \\
\text { management physician order as CPOE and apply it to } \\
\text { MOH hospitals and private sector } \\
\text { Review of Adults and Pediatrics pain management } \\
\text { guidelines utilization at MOH hospitals and private sector } \\
\text { Review Adults and Pediatrics pain management } \\
\text { medications consumption at MOH hospitals and private } \\
\text { sector } \\
\text { Delivery the Adults and Pediatrics pain management } \\
\text { training Courses at MOH and private sector } \\
\text { Delivery the Adults and Pediatrics pain management } \\
\text { training courses for twenty regions and private sector }\end{array}$ \\
\hline
\end{tabular}

Table 3: Hospital Pain Management Program Key Performance Indicators.

\begin{tabular}{|c|c|c|c|c|c|c|}
\hline \multicolumn{7}{|c|}{ Hospital Pain Management Program Key Performance Indicators } \\
\hline No & Indicator Name & Indicator Description & Indicator Formula (if exists) & Target & $\begin{array}{l}\text { Indicator } \\
\text { Frequency }\end{array}$ & $\begin{array}{l}\text { Example of } \\
\text { Compliance } \\
\text { (2014) }\end{array}$ \\
\hline \multicolumn{7}{|c|}{ Leadership and Management } \\
\hline 1 & $\begin{array}{l}\text { Central Pain } \\
\text { Management } \\
\text { Committee }\end{array}$ & $\begin{array}{l}\text { \% Pain Management } \\
\text { Committee meeting }\end{array}$ & $\begin{array}{l}\text { No Pain Management Committee } \\
\text { meeting/Total number of months }\end{array}$ & 1 & Annually & 1 \\
\hline 2 & $\begin{array}{l}\text { Regional Pain } \\
\text { Management } \\
\text { Committee }\end{array}$ & $\begin{array}{l}\text { \% Pain Management } \\
\text { Committee meeting }\end{array}$ & $\begin{array}{l}\text { No Pain Management Committee } \\
\text { meeting/Total number of months }\end{array}$ & 20 & Annually & 1 \\
\hline 3 & $\begin{array}{l}\text { Hospital Pain } \\
\text { Management } \\
\text { Committee }\end{array}$ & $\begin{array}{l}\text { \% Pain Management } \\
\text { Committee meeting }\end{array}$ & $\begin{array}{l}\text { No Pain Management Committee } \\
\text { meeting/Total number of months }\end{array}$ & 270 & Annually & 10 \\
\hline 4 & $\begin{array}{c}\text { Central Pain } \\
\text { Management } \\
\text { Committee meeting }\end{array}$ & $\begin{array}{l}\text { \% Pain Management } \\
\text { Committee meeting }\end{array}$ & $\begin{array}{l}\text { No Pain Management Committee } \\
\text { meeting/Total number of months }\end{array}$ & 12 & Annually & 10 \\
\hline 5 & $\begin{array}{c}\text { Regional Pain } \\
\text { Management } \\
\text { Committee meeting }\end{array}$ & $\begin{array}{l}\text { \% Pain Management } \\
\text { Committee meeting }\end{array}$ & $\begin{array}{l}\text { No Pain Management Committee } \\
\text { meeting/Total number of months }\end{array}$ & 240 & Annually & 12 \\
\hline 6 & $\begin{array}{l}\text { Hospital Pain } \\
\text { Management } \\
\text { Committee }\end{array}$ & $\begin{array}{l}\text { \% Pain Management } \\
\text { Committee meeting }\end{array}$ & $\begin{array}{l}\text { No Pain Management Committee } \\
\text { meeting/Total number of months }\end{array}$ & 3240 & Annually & 120 \\
\hline 7 & $\begin{array}{c}\text { Pain Management } \\
\text { Physician order Manual } \\
\text { Booklet }\end{array}$ & $\begin{array}{c}\text { \% Update of pain } \\
\text { management guidelines } \\
\text { as physician order sheet } \\
\text { for acute and chronic pain } \\
\text { printed }\end{array}$ & $\begin{array}{l}\text { No Pain Management Booklet printed/ } \\
\text { Total number of hospitals distributed }\end{array}$ & 270 & Annually & 10 \\
\hline 8 & $\begin{array}{l}\text { Pain Management } \\
\text { Manual Electronic }\end{array}$ & $\begin{array}{c}\text { \% Update of pain } \\
\text { management guidelines } \\
\text { as physician order sheet } \\
\text { for acute and chronic pain } \\
\text { during CPOE }\end{array}$ & $\begin{array}{l}\text { No Pain Management as CPOE /Total } \\
\text { number of hospitals had CPOE }\end{array}$ & 50 & Annually & NA \\
\hline \multicolumn{7}{|c|}{ Optimal Pain Management Use } \\
\hline 1 & $\begin{array}{c}\text { Medication } \\
\text { reconciliation ER of } \\
\text { painkiller medications }\end{array}$ & $\begin{array}{l}\% \text { of Medication } \\
\text { reconciliation discrepancy } \\
\text { at ER }\end{array}$ & $\begin{array}{l}\text { No of patient of painkiller medications } \\
\text { discrepancy/ total no medication of } \\
\text { reconciliation during ER visit }\end{array}$ & 0 & Monthly & NA \\
\hline 2 & $\begin{array}{l}\text { Medication } \\
\text { reconciliation During } \\
\text { transferring from unit } \\
\text { to unit of painkiller } \\
\text { medications }\end{array}$ & $\begin{array}{l}\% \text { of Medication } \\
\text { reconciliation discrepancy } \\
\text { During transferring from } \\
\text { unit to unit }\end{array}$ & $\begin{array}{c}\text { No of patient of painkiller medications } \\
\text { discrepancyreconciliation during } \\
\text { transferring / total no of medicines } \\
\text { transferring patients }\end{array}$ & 0 & Monthly & NA \\
\hline
\end{tabular}




\begin{tabular}{|c|c|c|c|c|c|c|}
\hline 3 & $\begin{array}{c}\text { Medication } \\
\text { reconciliation During } \\
\text { discharge of painkiller } \\
\text { medications }\end{array}$ & $\begin{array}{l}\text { \% of Medication discrepancy } \\
\text { reconciliation During } \\
\text { discharge of painkiller } \\
\text { medications }\end{array}$ & $\begin{array}{c}\text { No of patient of painkiller medications } \\
\text { discrepancyreconciliation during } \\
\text { discharge / total no of drugs discharge } \\
\text { patients }\end{array}$ & 0 & Monthly & NA \\
\hline 4 & $\begin{array}{l}\text { Drug-related hospital } \\
\text { admission of painkiller } \\
\text { medications. }\end{array}$ & $\begin{array}{l}\% \text { of drug-related hospital } \\
\text { admission }\end{array}$ & $\begin{array}{c}\text { No patient admitted to due hospital to } \\
\text { drug related problem / total number } \\
\text { of admission }\end{array}$ & 0 & Monthly & NA \\
\hline 5 & $\begin{array}{l}\text { Drug-related hospital } \\
\text { ER visit of painkiller } \\
\text { medications. }\end{array}$ & $\begin{array}{c}\text { \% Drug related hospital ER } \\
\text { visit }\end{array}$ & $\begin{array}{c}\text { No patient visited ER hospital due } \\
\text { drug related problem / total number } \\
\text { of admission }\end{array}$ & 0 & Monthly & NA \\
\hline 6 & $\begin{array}{l}\text { Someunapproved drug } \\
\text { indication painkiller } \\
\text { medications. }\end{array}$ & $\begin{array}{l}\% \text { drug prescribed by } \\
\text { physician for unapproved } \\
\text { medication }\end{array}$ & $\begin{array}{c}\text { Number of unapproved drug } \\
\text { indication per } 100 \text { patient receiving } \\
\text { pain management services } \\
\text { Number of drug unapproved } \\
\text { indication/ Total number } \\
\text { prescriptions of pain management } \\
\text { services }\end{array}$ & 0 & Quarterly & NA \\
\hline 7 & $\begin{array}{l}\text { Automatic Stop Order } \\
\text { (ASO) of painkiller } \\
\text { medications }\end{array}$ & $\begin{array}{l}\% \text { Automatic Stop Order } \\
\text { (ASO) painkiller medications } \\
\text { through drug distribution } \\
\text { system (unit dose system) }\end{array}$ & $\begin{array}{c}\text { No. of Automatic Stop Order (ASO) } \\
\text { painkiller medications / total number } \\
\text { of patient receiving painkiller } \\
\text { medications }\end{array}$ & 100 & Monthly & Not reported \\
\hline 8 & $\begin{array}{l}\text { Pharmacist intervention } \\
\text { of painkiller } \\
\text { medications }\end{array}$ & $\begin{array}{l}\text { No. of intervention or any } \\
\text { suggestions by Pharmacist of } \\
\text { painkiller medications }\end{array}$ & $\begin{array}{c}\text { No. of pharmacist intervention per } \\
100 \text { patient receiving painkiller } \\
\text { medications }\end{array}$ & $\begin{array}{c}\text { Not } \\
\text { specific }\end{array}$ & Monthly & Not reported \\
\hline 9 & $\begin{array}{l}\text { Patient Satisfaction Pain } \\
\text { management guidelines }\end{array}$ & $\begin{array}{l}\% \text { Patient Satisfaction Pain } \\
\text { management guidelines }\end{array}$ & $\begin{array}{l}\text { No patient satisfied receiving } \\
\text { painkiller medications guidelines } \\
\text { / Total number patient receiving } \\
\text { painkiller medications }\end{array}$ & $100 \%$ & Monthly & NA \\
\hline 10 & $\begin{array}{l}\text { Patient Pain } \\
\text { Management Patient } \\
\text { Quality of Life }\end{array}$ & $\begin{array}{l}\text { \% Patient Pain Management } \\
\text { Patient Quality of Life }\end{array}$ & $\begin{array}{c}\text { No patient Quality of Life receiving } \\
\text { painkiller medications guidelines } \\
\text { / Total number patient pain } \\
\text { management }\end{array}$ & $100 \%$ & Monthly & NA \\
\hline 11 & $\begin{array}{l}\text { Patient counseling Pain } \\
\text { management guidelines }\end{array}$ & $\begin{array}{l}\% \text { Patient counseling Pain } \\
\text { management guidelines }\end{array}$ & $\begin{array}{l}\text { No patient counseling receiving } \\
\text { painkiller medications guidelines / } \\
\text { Total number patient counseling }\end{array}$ & $100 \%$ & Monthly & Not reported \\
\hline 12 & $\begin{array}{l}\text { Non-available crash cart } \\
\text { medication of painkiller } \\
\text { medications }\end{array}$ & $\begin{array}{l}\% \text { of non-available crash cart } \\
\text { medication of painkiller } \\
\text { medications }\end{array}$ & $\begin{array}{l}\text { No of crash cart of painkiller } \\
\text { medications prescription not } \\
\text { dispensed / total crash cart of } \\
\text { painkiller medications prescription }\end{array}$ & 0 & Monthly & 0 \\
\hline 13 & $\begin{array}{l}\text { Antidote gave to } \\
\text { patient of painkiller } \\
\text { medications }\end{array}$ & $\begin{array}{l}\% \text { of Antidote given to } \\
\text { patient during poisoning }\end{array}$ & $\begin{array}{c}\text { Number of patients receiving } \\
\text { antidote of painkiller medications / } \\
\text { total number of patients supposed } \\
\text { to receive antidote of painkiller } \\
\text { medications }\end{array}$ & 0 & Monthly & 0 \\
\hline \multicolumn{7}{|c|}{ Monitoring Pain Management Medications and Use } \\
\hline 1 & $\begin{array}{l}\text { Non-available of acute } \\
\text { painkiller medications }\end{array}$ & $\begin{array}{l}\% \text { of non-available of acute } \\
\text { painkiller medications }\end{array}$ & $\begin{array}{c}\text { No of painkiller medications } \\
\text { prescription not dispensed / total } \\
\text { painkiller medications prescription }\end{array}$ & 0 & Monthly & 0 \\
\hline 2 & $\begin{array}{c}\text { Non-available } \\
\text { medication of chronic } \\
\text { pain killer medications. }\end{array}$ & $\begin{array}{l}\% \text { of non-available chronic } \\
\text { pain killer medications. }\end{array}$ & $\begin{array}{l}\text { No of medication dispensed from } \\
\text { medical supply / No of requested } \\
\text { medication from medical supply }\end{array}$ & 0 & Monthly & 0 \\
\hline 3 & $\begin{array}{l}\text { Non-available OPD } \\
\text { medication of painkiller } \\
\text { medications }\end{array}$ & $\begin{array}{l}\% \text { of non-available } \\
\text { medication in OPD }\end{array}$ & $\begin{array}{l}\text { No of OPD of painkiller medications } \\
\text { prescription dispensed / total OPD of } \\
\text { painkiller medications prescription }\end{array}$ & 0 & Monthly & 0 \\
\hline 4 & $\begin{array}{l}\text { Adherence to Acute Pain } \\
\text { management guidelines }\end{array}$ & $\begin{array}{l}\% \text { adherence to Pain } \\
\text { management guidelines }\end{array}$ & $\begin{array}{l}\text { No acute painkiller medications } \\
\text { adhere to guidelines / Total number } \\
\text { acute painkiller medications }\end{array}$ & $50 \%$ & Monthly & NA \\
\hline
\end{tabular}




\section{Journal of Pharmacology \& Clinical Research}

\begin{tabular}{|c|c|c|c|c|c|c|}
\hline 5 & $\begin{array}{c}\text { Adherence to } \\
\text { Chronic Pain } \\
\text { ManagementGuidelines }\end{array}$ & $\begin{array}{l}\% \text { adherence to Pain } \\
\text { management guidelines }\end{array}$ & $\begin{array}{l}\text { No Chronic painkiller medications } \\
\text { adhere to guidelines / Total number } \\
\text { Chronic painkiller medications }\end{array}$ & $50 \%$ & Monthly & NA \\
\hline 6 & $\begin{array}{l}\% \text { Drug Utilization of } \\
\text { painkiller medications }\end{array}$ & $\begin{array}{l}\text { Drug Utilization Evaluation } \\
\text { of expensive or high-risk } \\
\text { medication }\end{array}$ & $\begin{array}{c}\text { No Selected Drug Utilization adhere } \\
\text { to usage criteria / Total Selected } \\
\text { Drug Utilization to accede to all usage } \\
\text { criteria }\end{array}$ & $50 \%$ & $\begin{array}{l}\text { Quarterly } \\
\text { Bi-annually }\end{array}$ & NA \\
\hline 7 & $\begin{array}{l}\text { Medication Error } \\
\text { Report painkiller } \\
\text { medications. }\end{array}$ & Rate of Medication Errors & $\begin{array}{l}\text { No. of medication error per } 100 \\
\text { patient receiving pharmacy services } \\
\text { Number of medication errors per } \\
100 \text { days LOS Number of medication } \\
\text { errors/ number of prescription }\end{array}$ & 0 & Monthly & Not reported \\
\hline 8 & $\begin{array}{c}\text { Drug Quality Report } \\
\text { painkiller medications. }\end{array}$ & Rate of Drug Quality & $\begin{array}{l}\text { No. of Drug Quality discrepancy per } \\
100 \text { patient receiving pharmacy } \\
\text { services Number of Drug Quality } \\
\text { discrepancy per } 100 \text { days LOS Number } \\
\text { of Drug Quality discrepancy / number } \\
\text { of prescription }\end{array}$ & 0 & Monthly & Not reported \\
\hline 9 & $\begin{array}{l}\text { Adverse Drug Reaction } \\
\text { Report painkiller } \\
\text { medications. }\end{array}$ & $\begin{array}{l}\text { Rate of Adverse Drug } \\
\text { Reaction }\end{array}$ & $\begin{array}{l}\text { No. of adverse drug reaction per } 100 \\
\text { patient receiving pharmacy services } \\
\text { Number of adverse drug reaction per } \\
100 \text { days LOS Number of Adverse drug } \\
\text { reaction/number of prescription }\end{array}$ & 0 & Monthly & Not reported \\
\hline 10 & $\begin{array}{l}\text { Average wait time for a } \\
\text { prescription }\end{array}$ & $\begin{array}{c}\text { No of minutes of Patient } \\
\text { waiting time for prescription }\end{array}$ & $\begin{array}{l}\text { The time calculated when receiving } \\
\text { prescriptions until dispensing the } \\
\text { pain management medication to the } \\
\text { patients }\end{array}$ & $10-20$ & Monthly & NA \\
\hline 11 & $\begin{array}{l}\text { Self Assessment of } \\
\text { General of painkiller } \\
\text { medications safety }\end{array}$ & $\begin{array}{c}\text { International Safe } \\
\text { Medication Practice } \\
\text { assessment for all general } \\
\text { safety plan }\end{array}$ & Painkiller medications safety & 100 & $\begin{array}{l}\text { Every six } \\
\text { months }\end{array}$ & NA \\
\hline 12 & $\begin{array}{l}\text { Pain Management } \\
\text { Consumption }\end{array}$ & $\begin{array}{l}\% \text { Total budget of painkiller } \\
\text { medications }\end{array}$ & $\begin{array}{c}\text { No of painkiller medications budget } \\
\text { / No of total pharmacy medications } \\
\text { budget }\end{array}$ & $\begin{array}{l}\text { Not } \\
\text { specific }\end{array}$ & Annually & NA \\
\hline \multicolumn{7}{|c|}{ Education } \\
\hline 1 & $\begin{array}{l}\text { Pain Management } \\
\text { Course (Central) }\end{array}$ & \% Pain Management course & $\begin{array}{l}\text { No Pain Management course /Total } \\
\text { number of courses annually }\end{array}$ & 2 & Annually & 1 \\
\hline 2 & $\begin{array}{l}\text { Pain Management } \\
\text { Course (Region) }\end{array}$ & \% Pain Management course & $\begin{array}{l}\text { No Pain Management course /Total } \\
\text { number of courses annually }\end{array}$ & $2-4$ & Annually & NA \\
\hline 3 & $\begin{array}{l}\text { Pain Management } \\
\text { Course (Peripheral) }\end{array}$ & $\%$ Pain Management course & $\begin{array}{l}\text { No Pain Management course /Total } \\
\text { number of courses annually }\end{array}$ & 270 & Annually & NA \\
\hline \multicolumn{7}{|c|}{ Research } \\
\hline 1 & $\begin{array}{l}\text { Cost avoidance of } \\
\text { Pharmacist intervention } \\
\text { of pain killer } \\
\text { medications }\end{array}$ & $\begin{array}{l}\text { Estimate cost avoidance of } \\
\text { Pharmacist Intervention of } \\
\text { pain killer medications }\end{array}$ & $\begin{array}{l}\text { Total estimated Cost avoidance of } \\
\text { pharmacist intervention per month of } \\
\text { pain killer medications }\end{array}$ & $\begin{array}{l}10,000 \\
\text { USD }\end{array}$ & monthly & NA \\
\hline 2 & $\begin{array}{l}\text { Cost avoidance of } \\
\text { answering drug } \\
\text { information question of } \\
\text { pain killer medications }\end{array}$ & $\begin{array}{l}\text { No. of DPIC questions asked } \\
\text { by healthcare professionals }\end{array}$ & $\begin{array}{l}\text { Total estimated Cost avoidance of } \\
\text { answering drug information question } \\
\text { per month of painkiller medications }\end{array}$ & $\begin{array}{l}10,000 \\
\text { USD }\end{array}$ & monthly & NA \\
\hline 3 & $\begin{array}{l}\text { Incremental cost drug } \\
\text { related problem of } \\
\text { painkiller medications }\end{array}$ & $\begin{array}{l}\text { Additional charge of drug- } \\
\text { related problem of painkiller } \\
\text { medications }\end{array}$ & $\begin{array}{l}\text { Total estimated incremental LOS and } \\
\text { related issues due to drug related } \\
\text { problem, medication errors, adverse } \\
\text { drug reaction, untreated indication, } \\
\text { non-compliance, drug poisoning, of } \\
\text { painkiller medications }\end{array}$ & $\begin{array}{l}\text { Non- } \\
\text { specific }\end{array}$ & Monthly & NA \\
\hline
\end{tabular}




\section{Conclusion}

National pharmacy pain management program is critical system at Ministry of Health institutions in Saudi Arabia. The program is unique national wise and Gulf countries level. The implementation of the program prevents drug misuse, improve medication safety system, prevent medications errors, improve patient outcome and avoid unnecessary economic burden on health care organizations.

\section{References}

1. Kaki A M (2006) Pain clinic experience in teaching hospital in Western Saudi Arabia. Saudi Med J 27(12): pp. 1882-1886.

2. Anwari J S, Ahmed F, Mustafa T (2005) An audit of acute pain service in Central, Saudi Arabia. Saudi Med J 26(2): 298-305.

3. Alomi, YA (2016) National Drug Information Center Program at Ministry of Health in Saudi Arabia. Advances in Pharmacoepidemiology \& Drug Safety 05(1).

4. (2014) Joint Commission International Accreditation Standards for Hospitals. ( $5^{\text {th }}$ edn) Joint Commission Resources.

5. (2015) Saudi Central Board for Accreditation of Healthcare Institutions. National Hospital Standards. ( $3^{\text {rd }}$ edn). CBAHI Publications.

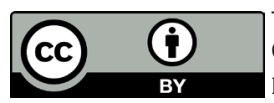

This work is licensed under Creative Commons Attribution 4.0 License

DOI: $10.19080 /$ JPCR.2017.03.555606
6. Al Rowaili A, Al ageel S, Al Naim L, Al Diab A (2009) Appropriateness of cancer pain management in Saudi teaching hospital. G.J.0 (5): 37-43.

7. Kaki A (2011) Medical students' knowledge and attitude toward cancer pain management in Saudi Arabia. Saudi Med J 32(6): 628-632.

8. Alsayadi S, Alzohaibi A (2014) Knowledge management implementation reality in Saudi ground services: an exploratory study. International Journal of Academic Research 6(3): 61-168.

9. Eid T, Manias E, Bucknall T, Almazrooa A (2014) Nurses' Knowledge and Attitudes Regarding Pain in Saudi Arabia. Pain Management Nursing 15(4): e25-e36.

10. (2016) About the Ministry - Strategy. Ministry of Health Portal, Kingdom of Saudi Arabia.

11. Alomi YA, Alghamdi SJ, Alattyh RA (2015) Strategic Plan of General Administration of Pharmaceutical Care at Ministry of Health in Saudi Arabia 2012 - 2022. J Pharm Pharm Scien 1(3): 1-8.

12. Alomi Y (2016) Pharmaceutical care strategic plan with pharmaceutical companies; future vision in Saudi Arabia.Journal of Developing Drugs 05(02).

13. Alomi YA (2015) National Pharmacy Practice Programs at Ministry of Health in Saudi Arabia. J Pharm Pharm Scien 1(2): 17-18.

\section{Your next submission with Juniper Publishers will reach you the below assets}

- Quality Editorial service

- Swift Peer Review

- Reprints availability

- E-prints Service

- Manuscript Podcast for convenient understanding

- Global attainment for your research

- Manuscript accessibility in different formats (Pdf, E-pub, Full Text, Audio)

- Unceasing customer service

Track the below URL for one-step submission https://juniperpublishers.com/online-submission.php 